\title{
Cervical expression of elafin and secretory leukocyte peptidase inhibitor does not predict preterm delivery in twin pregnancy - results from a pilot study
}

Aleksandra Saletra-Bielińska

1st Department of Obstetrics and Gynecology, First Faculty of Medicine, Medical University of Warsaw, Warsaw, Poland

(iD) https://orcid.org/0000-0003-1802-3334

\section{Katarzyna Kosińska-Kaczyńska}

Second Department of Obstetrics and Gynecology, The Center of Postgraduate Medical Education

(iD) https://orcid.org/0000-0001-5461-273X

Corresponding author:

katarzyna.kosinska-kaczynska@cmkp.edu.pl

Iwona Szymusik

1st Department of Obstetrics and Gynecology, First Faculty of Medicine, Medical University of Warsaw, Warsaw, Poland

(iD) https://orcid.org/0000-0001-8106-5428

\section{Justyna Niderla-Bielińska}

Department of Histology and Embryology, Center for Biostructure, Medical University of Warsaw

(iD) https://orcid.org/0000-0002-9295-480X

\section{Jacek Malejczyk}

Department of Histology and Embryology, Center for Biostructure, Medical University of Warsaw

(iD) https://orcid.org/0000-0002-5371-8476

Mirosław Wielgoś

1st Department of Obstetrics and Gynecology, First Faculty of Medicine, Medical University of Warsaw, Warsaw, Poland

(iD) https://orcid.org/0000-0003-2581-3668
DOI: https://doi.org/10.20883/medical.e490

Keywords: twin pregnancy, elafin, secretory leukocyte peptidase inhibitor, preterm delivery

Published: 2021-03-30

How to Cite: Saletra-Bielińska A, Kosińska-Kaczyńska K, Szymusik I, Niderla-Bielińska J, Malejczyk J, Wielgoś M. Cervical expression of elafin and secretory leukocyte peptidase inhibitor does not predict preterm delivery in twin pregnancy - results from a pilot study. JMS [Internet]. 2021 Feb 24;90(1):e490. doi:10.20883/medical.408

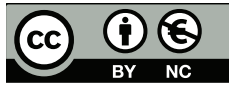

(C) 2021 by the author(s). This is an open access article distributed under the terms and conditions of the Creative Commons Attribution (CC BY-NC) licencse. Published by Poznan University of Medical Sciences

\begin{abstract}
Introduction. Elafin and secretory leukocyte peptidase inhibitor may serve as the predictors of cervical shortening and preterm delivery in twin gestation.

Material and Methods. A prospective observational study was conducted between September 2016 and March 2017. Cervicovaginal swabs collected from 40 women with twin gestation were analysed and the mRNA expression of elafin and secretory leukocyte peptidase inhibitor (SLPI) correlated with preterm delivery. Results. The mean gestational age at delivery was $35.6 \pm 5.8$ weeks, with 23 women delivering before 37 weeks (57.5\%), 7 before 34 weeks (17.5\%) and 3 before 32 weeks of gestation (7.5\%). The mRNA expression of elafin and SLPI was not dependent on chorionicity and did not correlate with gestational age at delivery.

Conclusions. Elafin and SLPI are not appropriate predictors of preterm delivery in twins.
\end{abstract}

\section{Introduction}

Elafin and secretory leukocyte peptidase inhibitor (SLPI) are the members of the antimicrobial peptide family, playing an important role in the modulation of the immune system. They are major protease inhibitors secreted at mucosal surfaces and the first line of defence against foreign antigens. They 
are expressed throughout the female genital tract, inhibiting bacterial, viral and fungal activity and present anti-protease activity on proteases induced by infection and inflammation on the epithelial surfaces [1]. They belong to the whey acidic protein (WAP) family, as SLPI has two WAP domains and elafin has one WAP domain, which serve as neutrophil elastase inhibitors. Elafin, also known as peptidase inhibitor 3 (PI3), is a $9.9 \mathrm{kDa}$ protein which is the C-terminal region of trappin-2. It inhibits neutrophil elastase and proteinase 3 . Elafin expression was reported to be elevated in fetal membranes of preterm prelabour rupture of membranes (PPROM) [2] and the cervicovaginal fluid of women delivering preterm in singleton gestation [3]. The PI3 gene produces a complex protein $(12.3 \mathrm{kDa})$ which is split intracellularly into the mature form (9.9 kDa) and secreted into the extracellular matrix and transformed into soluble elafin [3]. Its production is stimulated by lipopolysaccharide and inflammatory cytokines and decreased by oestradiol [3] SLPI is an $11.9 \mathrm{kDa}$ protein and an anti-inflammatory mediator [4]. Its N-terminal domain presents activity against both gram-positive and gram-negative organisms. The $\mathrm{C}$-terminal domain is a strong inhibitor of neutrophil elastase, cathepsin G, trypsin, chymotrypsin, tryptase, and chymase. SLPI negatively regulates proinflammatory signalling mediated by nuclear factor-kappa B (NF-kB) [4].

According to Romero, preterm delivery is a common symptom of various processes and causes [5], with infection being the etiologic factor in $30 \%$ of preterm deliveries in singleton pregnancies [5]. During gestation, the uterine cervix and its mucus play an important role in the protection from pathogens present in the vagina. If the barrier is insufficient, infection and inflammation trigger cervical remodelling and shortening which leads to delivery. Macrophages and neutrophils infiltrate the cervix and the local production of proteases begins tissue remodelling and cervical opening. Preterm delivery affects two-thirds of all twin pregnancies and contributes to $50 \%$ of all neonatal twin deaths [6]. In the Preterm Prediction Study, $54.4 \%$ of all twins were born before 37 weeks, $32 \%$ before 35 weeks and $8.8 \%$ before completing 32 weeks of gestation [7]. It is a major problem associated with multiples, so finding an efficient predictor of preterm delivery in twin gestation is of great importance. It was hypothesised that elafin and SLPI may serve as the predictors of cervical shortening and preterm delivery in twin gestation.

\section{Materials and Methods}

For this pilot study of biomarkers in the prediction of preterm delivery in twins, a prospective observational study was conducted in the 1st Department of Obstetrics and Gynaecology, Medical University of Warsaw between September 2016 and March 2017. The study was approved by the Ethics Committee of the Medical University of Warsaw and was conducted according to the Declaration of Helsinki.

The inclusion criteria were a twin pregnancy beyond $22+0$ weeks of gestation, chorionicity established and documented on the 1st trimester sonographic scan (two gestational sacs or the lambda sign for a dichorionic pregnancy; a single gestational sac or $\mathrm{T}$ sign for a monochorionic pregnancy), verified gestational age (GA), known GA at delivery, newborns birth weight (BW) and complete medical data on the pregnancy outcome and neonatal outcome. Pregnancies complicated by one or two foetal demises, genetic or major anatomical abnormalities, twin to twin transfusion syndrome (TTTS), twin anaemia-polycythaemia sequence (TAPS), twin reversed arterial perfusion syndrome (TRAP), as well as monochorionic monoamniotic ones, were excluded from the study. GA was calculated based on the first day of the last menstrual period or a transfer day in assisted reproductive technique procedures and verified by the crown-rump length (CRL) measured on the first trimester scan (if estimated due dates were inconsistent and the difference was over 5 days, the ultrasound measurement was of primary importance; in case of CRL discordance, the measurement from the larger twin was chosen). Body mass index (BMI) was defined as the body mass divided by the square of the body height. Preterm delivery was defined as the delivery occurring before completed 37 weeks. PPROM was defined as amniotic fluid leakage before 37 weeks of gestation, without spontaneous uterine contractions. Gestational hypertension $(\mathrm{GH})$ and preeclampsia $(\mathrm{PE})$ were diagnosed according to American College of Obstetricians and Gynaecologists recommendations [8], whereas gestational diabetes mellitus (GDM) was according to the Polish Society of Obstetricians and Gynaecologists recommendations [9]. 
Women with dichorionic pregnancies were routinely counselled once every 4 weeks and in monochorionic pregnancies once every 2 weeks, including an ultrasound scan. In cases with no pregnancy complications, both monochorionic diamniotic and dichorionic twins were delivered beyond 37 weeks of gestation according to the local policy. All the women were counselled between 20 and 24 weeks of gestation. A routine ultrasound scan assessing foetal biometry and anatomy was performed during that period as well as an ultrasound measurement of the length of the cervical canal. This was measured according to the Foetal Medicine Foundation recommendations - a transvaginal probe was placed in the anterior fornix of the vagina with an empty bladder and the linear distance between callipers placed at the internal and external cervical os was taken. Also, cervicovaginal swab samples were collected with a special kit between 20 and 24 gestational weeks.

The primary outcome of the study was delivery occurring before the completion of 37 weeks of gestation. Secondary outcomes included deliveries before 34 weeks of gestation.

The RNA was isolated with a PureLink ${ }^{\text {TM }}$ RNA Micro Scale Kit (ThermoFisher Scientific, MA, USA) according to the manufacturer's protocol. The quality and concentration of RNA were assessed with a NanoDrop spectrophotometer and reverse transcription was performed with a High Capacity RNA-to-cDNA Kit according to the manufacturer's protocol (Applied Biosystems). Gene expression was analysed by relative quantitation ( $R Q$ ) using a comparative CT assay. Explants stimulated with the proangiogenic cocktail for PE stimulation and cells from the control group no. 1 for C199 stimulation were used as calibrators. Realtime PCR was performed on an Abi Prism 7500 (Applied Biosystems) in 96-well optical plates, with each sample run in triplicate and supplied with an endogenous control (human GAPDH no. Hs02786624_g1). The TaqMan Expression Assays (Applied Biosystems) SLPI: Hs00268204_m1 and elafin (PI3): Hs00964384_g1 were used and all probes were stained with FAM. Reactions were run in a $20 \mu$ l volume with TaqMan Universal Master Mix (Applied Biosystems), appropriate primer set, MGB probe and $5 \mathrm{ng}$ of cDNA template and universal thermal conditions were used, i.e. 10 min at $95^{\circ} \mathrm{C}, 40$ cycles of $15 \mathrm{~s}$ at $95^{\circ} \mathrm{C}$ and $1 \mathrm{~min}$ at $60^{\circ} \mathrm{C}$. Data analysis was performed with sequence detection software version 1.2 (Applied Biosystems, ThermoFisher Scientific, MA, USA).

Data are presented as the mean $( \pm S D)$, median or percentage. The Mann-Whitney test and Fisher's exact test were used for statistical analysis and a P-value $<0.05$ was considered significant. The sensitivity, specificity, positive predictive value, negative predictive value and positive and negative likelihood ratio with $95 \%$ confidence intervals were calculated to test the predictive value for preterm delivery (before 34 and 37 weeks of gestation). The data were analysed using Statistica version 13.1. Test performance was described for the prediction of delivery before 37- and 34-weeks using receiver operating characteristic (ROC) curves, sensitivity, specificity and predictive values. Areas under the ROC curve (AUC) were calculated and compared. Correlations between biomarkers and the cervical length were assessed by the Spearman rank correlation test.

\section{Results}

This pilot study involved 48 women. One case of an intrauterine foetal demise was diagnosed and one premature rupture of membranes occurred at 21 weeks of gestation; they were excluded from further analysis. Six patients were lost to followup, finally, 40 patients were deemed eligible for analysis.

The cervicovaginal swabs collected from 40 women with twin gestation were analysed and their basic characteristics are presented in Table 1. A cervical length below $25 \mathrm{~mm}$ between 20 and 24 weeks of gestation was diagnosed in three women and they were administered progesterone vaginally (200 mg per day). No vaginal pessary or cervical cerclage were administered.

The median GA at delivery was 36 weeks (interquartile range $35-37$ ), with 23 women delivering before 37 weeks (57.5\%), 7 before 34 weeks (17.5\%) and 3 before 32 weeks of gestation (7.5\%). All patients gave birth beyond 30 weeks. Preterm delivery was spontaneous due to PPROM or regular uterine contractions in 16 women (11 monochorionic diamniotic vs. 5 dichorionic pregnancies; $p=0.7$ ) and $82.5 \%$ of women had a caesarean delivery. The indications for caesarean section were malpresentation of the first foetus (6 monochorionic vs. 3 dichorionic twins), inter- 
twin growth discordance (2 vs. 1 respectively), PE ( 1 vs. 0 respectively) and maternal indications (2 vs. 1 respectively).

SLPI and elafin mRNA were detected in the cervical epithelial cells in all women and mRNA expression was not dependent on chorionicity (Table 1). There was no correlation between GA at delivery and the mRNA expression of SLPI or elafin. A separate analysis for the subgroup of women with spontaneous preterm delivery was performed, showing no relationship with biomarker expression [delta Ct SLP median 4.3, interquartile range (IQR) 3-4.9 in spontaneous preterm delivery groups vs. $3.4,2.7-3.6, p=0.2$; delta $\mathrm{Ct} \mathrm{PI} 32.6,1.8-3.5$ vs. 3.0, 2.3-3.5, respectively, $\mathrm{p}=0.5$ ]. Women delivering beyond 37 weeks had a significantly longer cervix assessed by ultrasound. No significant correlation between cervical length and biomarker expression was observed (delta Ct SLP: Spearman rank correlation coefficient $-0.08, p=0.6$; delta Ct PI3: Spearman rank correlation coefficient $-0.07, p=0.7$ ).

The cut-off points for PI3 and SLPI mRNA were designated based on the ROC curves and are presented in Figures 1A-D. The AUC for both elafin and SLPI was similar ranging from 0.53 to 0.602 . SLPI and PI3 had moderate sensitivity with low specificity in predicting preterm delivery before 37 and 34 weeks of gestation. The ROC curve was used to establish the cut-off point for cervical length in the prediction of preterm delivery, with a cervical length of $39 \mathrm{~mm}$ having sensitivity 561 (95\% Cl 32.2-.81.3), specificity 86.4 (95\% Cl 56.897.2), positive likelihood ratio $3.94(95 \% \mathrm{Cl} 1.3-12.2)$ and negative likelihood ratio $0.51(95 \% \mathrm{Cl} 0.29-$ $0.91)$. There were no significant differences in the expression of SLPI mRNA (median 4.2, IQR 3.1-5.1 vs. 3.3, 2.4-4.4 respectively; $p=0.3$ ) or expression of PI3 mRNA (3.1, $1.6-3.8$ vs. $2.8,2.5-3.2 ; \mathrm{p}=0.9$ ) between women who had cervical length below or above $39 \mathrm{~mm}$. The sensitivity, specificity, positive and negative likelihood ratios for delivery before 34 and 37 weeks, as well as cervical length less than $39 \mathrm{~mm}$, are shown in Table 2.

\section{Discussion}

This is the first study of elafin and SLPI expression in the cervicovaginal fluid in twin gestation, demonstrating no significant differences in elafin and a)

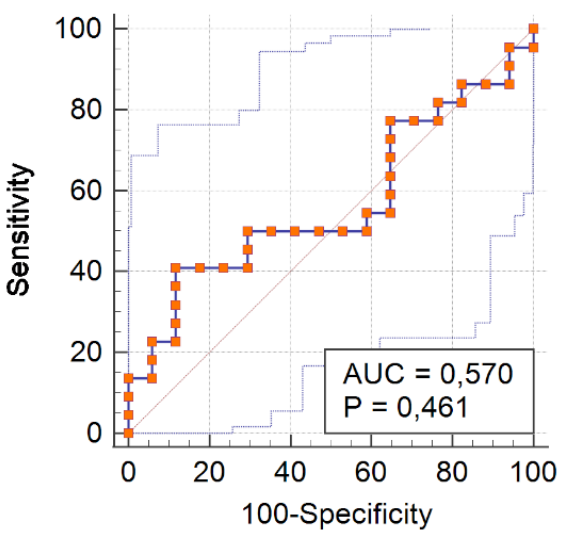

b)

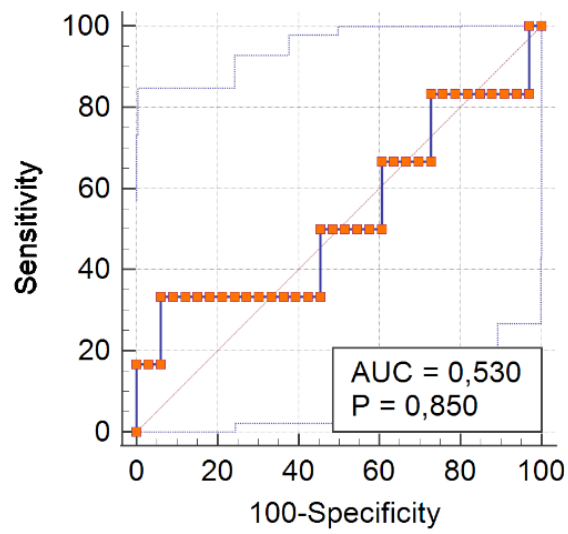

c)

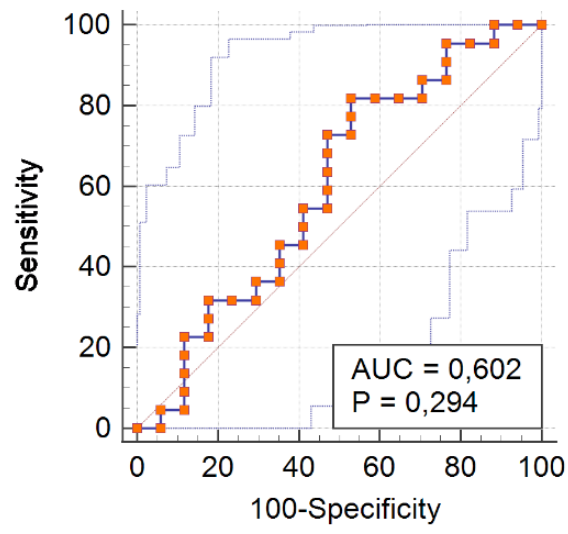

d)

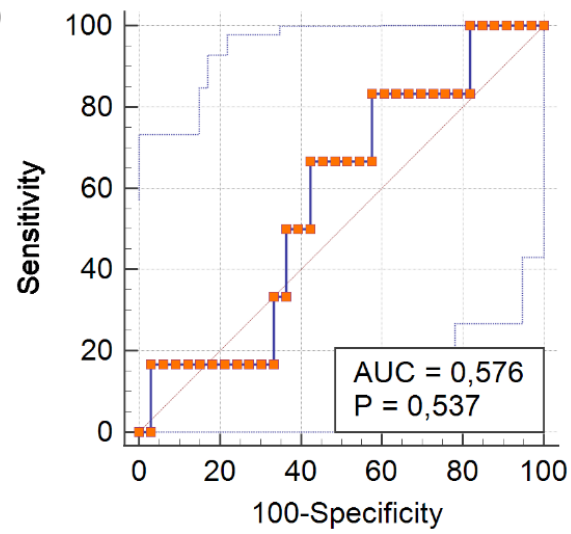

Figure 1. ROC curves demonstrating the ability of elafin and SLP cervicovaginal fluid to predict delivery before 37 and 34 weeks of gestation in twins. a) elafin in delivery prediction <37 weeks; b) elafin in delivery prediction <34 weeks; c) SLPI in delivery prediction <37 weeks; d) SLPI in delivery prediction $<34$ weeks 


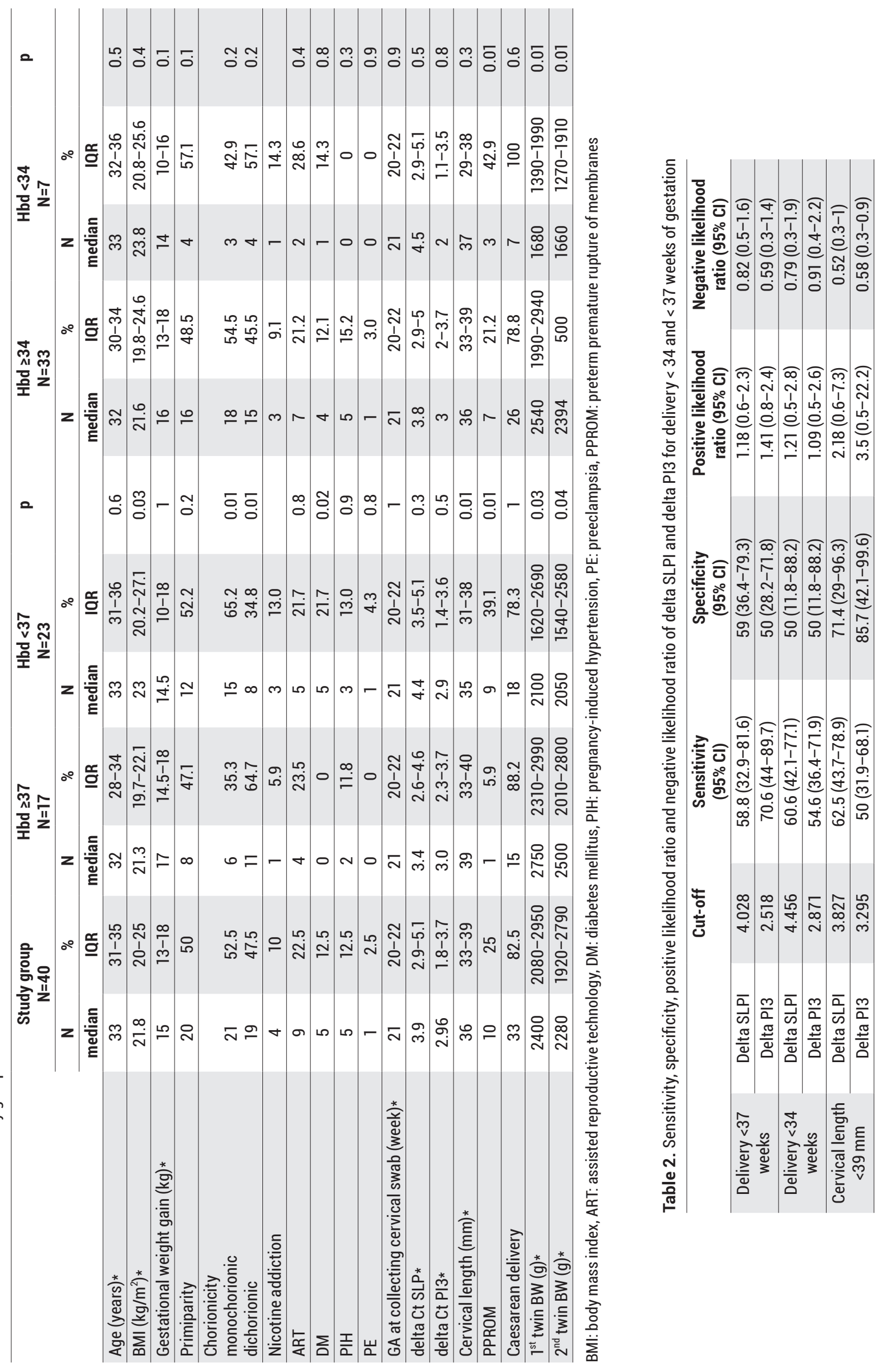


SLPI mRNA expression in the cervical fluid collected in mid-pregnancy between term and preterm deliveries. Elafin and SLPI had low specificity and moderate sensitivity in the prediction of delivery before 37 and 34 weeks, which is in line with other studies conducted in singleton pregnancies. Manning et al. detected no differences in elafin concentration in the cervicovaginal fluid of women delivering prior to or beyond 37 weeks in a study of 135 women with a history of preterm delivery (PTD) or cervical surgery [10]. Hezelgrave et al. observed 405 women with a singleton pregnancy at a high risk of PTD and 214 women at low risk of PTD, showing that elafin was not increased in high risk women who developed cervical shortening and delivered prematurely. The AUC for the prediction of delivery before 37 weeks was $0.52(0.44-$ $0.59)$ and before 34 weeks was $0.64(0.58-0.71)$ [11]. Another observational study of 104 singleton high risk pregnancies by Bastek et al. reported that elafin measured in the cervicovaginal fluid in midgestation was not a predictor of PTD [12].

Conversely, studies have confirmed a correlation between elafin expression and PTD. Itaoka et al. compared the mRNA expression of elafin in cervical swabs collected at 29 weeks of gestation in women at low and high risk of PTD, finding that the cervical mRNA expression of elafin was significantly higher in high risk women delivering preterm compared with high risk women delivering at term and low risk controls [13]. Similarly, Abbott et al. analysed the cervicovaginal fluid samples collected between 13 and 30 weeks of gestation of 74 asymptomatic women with a singleton gestation, showing that women who developed a short cervix had elafin concentrations 2.71 times higher than those who did not (Cl 1.94-3.79, $p=0.0005)$. Elafin concentrations were 3 -fold higher than in the controls when cervical shortening was first detected. Elafin in the cervicovaginal fluid collected before 24 weeks of gestation was significantly higher in women who had a spontaneous PTD (OR 1.79; $\mathrm{Cl}: 1.05-3.05, \mathrm{p}=0.034$ ) and elafin measured between $14+0-14+6$ weeks of pregnancy was predictive of subsequent development of a short cervix (AUC 1.00, $p=0.008$ ) within 8 weeks [3].

Similar findings concerning SLPI were published by Itaoka et al, who reported that the mRNA expression of SLPI in the cervicovaginal fluid was significantly higher in high risk women who delivered preterm than in low risk women, as well as in high risk women who delivered at term [13]. Conversely, Samejima et al. found no differences in SLPI concentrations between women delivering before and beyond 37 weeks gestation. However, they reported a significant correlation between cervical mucus SLPI concentration and inflammatory cytokines, such as IL- 6 and IL- 8 , which play an important role in preterm delivery [4].

In the present study, elafin and SLPI mRNA were detected in all collected samples. Itaoka et al. detected elafin and SLPI mRNA throughout the gestation and postpartum period, with the elafin concentration unchanged throughout pregnancy, while higher levels were detected after delivery [13]. In contrast, the expression of SLPI mRNA was the lowest in the first trimester, increasing in the second and third trimester [13]. Elafin and SLPI were also detected in the cervicovaginal secretion by other authors [14,15]. Differences in elafin and SLP production are probably controlled by hormones, especially progesterone. It was demonstrated that progesterone exposure increased SLPI but not elafin mRNA expression in a breast epithelial cell line [16], which may explain the differences in the observed expression of these antimicrobial peptides during pregnancy.

As uncomplicated twin pregnancies delivery was scheduled beyond 37 weeks in both dichorionic and monochorionic diamniotic gestation according to our local policy, it is assumed that the comparison of preterm deliveries between both groups is reliable. There was no correlation between elafin or SLPI and preterm delivery in twin gestation, which may be due to the small study group size, as this was a pilot study of potential biomarkers in the prediction of preterm delivery in twins. However, the aetiology of preterm delivery in twin gestation is complex and may differ from that in a singleton pregnancy. In twins, the overdistension of the uterus plays a major role and maybe a triggering factor for preterm delivery, even in the absence of infection and inflammation. The larger uterine cavity in the case of multiple pregnancies causes overdistension, which increases the expression of gap junctions in the myometrium and oxytocin receptors, as well as increased prostaglandin production [17]. As the aetiology of preterm delivery may be different from a singleton delivery, elafin and SLPI may not play a significant role in preterm delivery in multiple gestation. 
The elafin and SLPI production were assessed in this study by examining the expression of their mRNA rather than protein, as neutrophil elastase can interfere with the secretion of SLPI by forming a positively charged molecular complex with SLPI [13], thereby influences the measurement of protein concentration and induce bias. Hence, we considered mRNA expression to be more independent and objectively reflects protein production.

In conclusion, elafin and SLPI expression in the cervicovaginal fluid is not related to the risk of preterm delivery and not appropriate for the prediction of preterm delivery in twins.

\section{Acknowledgements}

\section{Conflict of interest statement}

The authors declare no conflict of interest.

\section{Funding sources}

There are no sources of funding to declare.

\section{References}

1. Sallenave J. Secretory Leukocyte Protease Inhibitor and Elafin/Trappin-2. American Journal of Respiratory Cell and Molecular Biology. 2010 Jun;42(6):635643. https://doi.org/10.1165/rcmb.2010-0095rt

2. Tromp G, Kuivaniemi $H$, Romero R, Chaiworapongsa T, Kim YM, Kim MR, Maymon E, Edwin S. Genomewide expression profiling of fetal membranes reveals a deficient expression of proteinase inhibitor 3 in premature rupture of membranes. American Journal of Obstetrics and Gynecology. 2004 Oct;191(4):13311338. https://doi.org/10.1016/j.ajog.2004.07.010

3. Abbott DS, Chin-Smith EC, Seed PT, Chandiramani M, Shennan AH, Tribe RM. Raised Trappin2/elafin Protein in Cervico-Vaginal Fluid Is a Potential Predictor of Cervical Shortening and Spontaneous Preterm Birth. Landay A. PLoS ONE. 2014 Jul 30;9(7):e100771. https://doi.org/10.1371/journal.pone.0100771

4. Samejima T, Nagamatsu T, Akiba N, Fujii T, Sayama S, Kawana K, Taguchi A, Kumasawa K, Iriyama T, Osuga Y, Fujii T. Secretory leukocyte protease inhibitor and progranulin as possible regulators of cervical remodeling in pregnancy. Journal of Reproductive Immunology. 2021 Feb;143:103241. https://doi. org/10.1016/j.jri.2020.103241

5. Romero R, Espinoza J, Gonçalves L, Kusanovic J, Friel L, Hassan S. The Role of Inflammation and Infection in Preterm Birth. Seminars in Reproductive Medicine. 2007 Jan;25(1):21-39. https://doi.org/10.1055/ s-2006-956773

6. Tarter JG, Khoury A, Barton JR, Jacques DL, Sibai BM. Demographic and obstetric factors influencing pregnancy outcome in twin gestations. American Journal of Obstetrics and Gynecology. 2002 May;186(5):910912. https://doi.org/10.1067/mob.2002.123989

7. Goldenberg RL, lams JD, Miodovnik M, Van Dorsten J, Thurnau G, Bottoms S, Mercer BM, Meis PJ, Moa- wad AH, Das A, Caritis SN, McNellis D. The preterm prediction study: Risk factors in twin gestations. American Journal of Obstetrics and Gynecology. 1996 Oct;175(4):1047-1053. https://doi.org/10.1016/ s0002-9378(96)80051-2

8. ACOG. Obstetrics \& Gynecology. 2019 Jan;133(1):1-1. https://doi.org/10.1097/aog.0000000000003018

9. Wender-Ożegowska E, Bomba-Opoń D, Brązert J, Celewicz Z, Czajkowski K, Gutaj P, Malinowska-Polubiec A, Zawiejska A, Wielgoś M. Standards of Polish Society of Gynecologists and Obstetricians in management of women with diabetes. Ginekol Pol. 2018;89(6):341-50. https://doi.org/10.5603/ GP.a2018.0059 PMID 30010185

10. Manning R, James CP, Smith MC, Innes BA, Stamp E, Peebles D, Bajaj-Elliott M, Klein N, Bulmer JN, Robson SC, Lash GE. Predictive value of cervical cytokine, antimicrobial and microflora levels for pre-term birth in high-risk women. Scientific Reports. 2019 Aug 2;9(1). https://doi.org/10.1038/s41598-019-47756-7

11. Hezelgrave NL, Seed $P T$, Chin-Smith EC, Ridout $A E$, Shennan AH, Tribe RM. Cervicovaginal natural antimicrobial expression in pregnancy and association with spontaneous preterm birth. Scientific Reports. 2020 Jul 21;10(1). https://doi.org/10.1038/s41598020-68329-z

12. Bastek JA, Hirshberg A, Chandrasekaran S, Owen CM, Heiser LM, Araujo BA, McShea MA, Ryan ME, Elovitz MA. Biomarkers and Cervical Length to Predict Spontaneous Preterm Birth in Asymptomatic High-Risk Women. Obstetrics \& Gynecology. 2013 Aug;122(2):283-289. https://doi.org/10.1097/ aog.0b013e31829ab714

13. Itaoka N, Nagamatsu T, Schust DJ, Ichikawa M, Sayama S, Iwasawa-Kawai Y, Kawana K, Yamashita T, Osuga Y, Fujii T. Cervical Expression of Elafin and SLPI in Pregnancy and Their Association With Preterm Labor. American Journal of Reproductive Immunology. 2015 Jan 5;73(6):536-544. https://doi. org/10.1111/aji.12354

14. Stock SJ, Duthie L, Tremaine T, Calder AA, Kelly RW, Riley SC. Elafin (SKALP/Trappin-2/proteinase inhibitor-3) Is Produced by the Cervix in Pregnancy and Cervicovaginal Levels Are Diminished in Bacterial Vaginosis. Reproductive Sciences. 2009 Sep;16(12):11251134. https://doi.org/10.1177/1933719109341998

15. Helmig R, Uldbjerg N, Ohlsson K. Secretory leukocyte protease inhibitor in the cervical mucus and in the fetal membranes. European Journal of Obstetrics \& Gynecology and Reproductive Biology. 1995 Mar;59(1):95-101. https://doi.org/10.1016/00282243(94)02023-8

16. King AE, Morgan K, Sallenave J, Kelly RW. Differential regulation of secretory leukocyte protease inhibitor and elafin by progesterone. Biochemical and Biophysical Research Communications. 2003 Oct;310(2):594-599. https://doi.org/10.1016/j. bbrc. 2003.08.151

17. Stock S, Norman J. Preterm and term labour in multiple pregnancies. Seminars in Fetal and Neonatal Medicine. 2010 Dec;15(6):336-341. https://doi. org/10.1016/j.siny.2010.06.006 